SISTEMA
ELETRÔNICO
DE REVISTAS
SER I UfPR

\title{
Panorama das Unidades de Conservação na zona costeira e marinha do estado de São Paulo
}

\section{Survey of Coastal and Marine Protected Areas in São Paulo State}

\author{
Evelin Eugênia de SOUSA ${ }^{1 *}$, Thiago Zagonel SERAFINI ${ }^{1}$ \\ ${ }^{1}$ Departamento de Ciências do Mar (DCMar), Universidade Federal de São Paulo (UNIFESP), Santos, SP, Brasil. \\ *E-mail de contato: evesp1997@hotmail.com
}

Artigo recebido em 7 de setembro de 2017, versão final aceita em 22 de janeiro de 2018.

RESUMO: O Sistema Nacional de Unidades de Conservação incorporou e adicionou categorias de áreas protegidas, ampliando as estratégias de conservação da natureza sob a tipologia de Unidade de Conservação (UC). No presente trabalho, foi realizado um levantamento das UCs criadas na zona costeira e marinha do estado de São Paulo, por meio de consultas a documentos, sites e bancos de dados governamentais. Foram levantadas 74 UCs federais, estaduais e municipais, sendo a maior parte resultante uma ação política estadual, com $69 \%$ das UCs sob sua gestão. Até o início dos anos de 1980 predominava a categoria Parque e, a partir de 2008, as UCs de uso sustentável superam em número e área as de proteção integral. Cerca de metade (53\%) das UCs conta com conselho gestor, instituído em um prazo médio de 5,2 anos, e 19\% contam com plano de manejo, instituído em média 21,6 anos após a sua criação. A criação de Mosaicos de UCs na zona costeira paulista se destaca, concentrando $25 \%$ de todos os mosaicos brasileiros, sendo que $59 \%$ das UCs fazem parte de ao menos um Mosaico. Atualmente as UCs são expressivas em todos os setores da zona costeira paulista, tornando-as um dos principais instrumentos de gestão costeira em São Paulo. A implementação de seus instrumentos de gestão e sua integração com outras políticas públicas, especialmente do gerenciamento costeiro, possibilitaria a inserção mais efetiva das UCs nas dinâmicas territoriais da zona costeira paulista.

Palavras-chave: áreas protegidas; conservação da natureza; Sistema Nacional de Unidades de Conservação; gestão costeira; São Paulo.

ABSTRACT: The Brazilian National System of Protected Areas incorporated and added categories of Protected Areas (PA), expanding the strategies of nature conservation. In the present study, a survey of the PAs implemented in this region was carried out, through consultations with government documents, websites and databases. Seventyfour federal, state and municipal PAs were surveyed, most of which resulted in a state political action, with 
$69 \%$ of the PAs under its management. Until the early 1980s, the Parks were predominant and since 2008 PA of sustainable use have outnumbered and in area PA of integral protection. About half $(53 \%)$ of the PA have an advice board, which is set up in an average period of 5.2 years, and 19\% have a management plan, which was implemented about 21.6 years after its creation. The creation of PA Networks (Mosaics) in São Paulo's coastal zone stands out, concentrating $25 \%$ of all Brazilian Mosaics, with $59 \%$ of PAs being part of at least one of them. Currently, PAs are significant in all sectors of the coastal zone, making them one of the main coastal management tools in São Paulo. Its integration with other public policies, especially of coastal management, would allow a more effective insertion of PAs into the territorial dynamics of São Paulo's coastal zone.

Keywords: protected area; nature conservation; Brazilian National System of Protected Areas; coastal management; São Paulo.

\section{Introdução}

A criação de áreas protegidas vem sendo a principal estratégia adotada globalmente para a conservação da natureza (Edgar et al., 2014). No Brasil, as políticas públicas ambientais de regularização do uso dos recursos naturais iniciaram sobretudo a partir dos anos de 1930, com a criação de diferentes instrumentos legais, os quais previam áreas especialmente protegidas, resultando, em 2000, em um sistema integrado (o Sistema Nacional de Unidades de Conservação - SNUC) (Medeiros, 2006; Peccatiello, 2011). Atualmente, o SNUC regulamenta uma das tipologias de áreas protegidas no Brasil, as Unidades de Conservação (UCs ${ }^{1}$ ), correspondendo à principal estratégia de gestão e conservação da biodiversidade no país (Silva, 2005).

O SNUC é resultado de um longo processo de embates entre diferentes interpretações da questão ambiental no país, representadas por perspectivas preservacionistas, conservacionistas, socioambientalistas e ruralistas, muitas delas contraditórias entre si (Peccatiello, 2011). Este processo engendrou um sistema que abarca diferentes possibilidades de conservação da natureza a partir de 12 categorias de UCs distribuídas em dois grupos, que se diferenciam pelo potencial de uso dos recursos naturais, de forma indireta (grupo de Proteção Integral) ou direta (grupo de Uso Sustentável) ${ }^{2}$. A efetividade dessas diversas categorias em de fato possibilitar a conservação da biodiversidade ainda é motivo de discussão entre diferentes visões sobre a conservação, de maneira geral, opondo perspectivas preservacionistas e socioambientalistas (Dourojeanni \& Pádua, 2007; Pereira \& Diegues, 2010).

A zona costeira do estado de São Paulo mantém parte considerável de seus ecossistemas relativamente conservados (SMA, 2015), assim como abriga historicamente populações tradicionais, principalmente de origem Caiçara (Ferreira, 2004). Em

\footnotetext{
${ }^{1}$ A terminologia Unidade de Conservação (UC) somente é adotada a partir da criação do SNUC em 2000, referindo-se a uma tipologia de Área Protegida no Brasil. Com o SNUC, diversas categorias de Áreas Protegidas regulamentadas por diferentes dispositivos legais passaram a integrar o conjunto de categorias previstas no SNUC, adotando a terminologia de UC.

${ }^{2}$ Pertencem ao grupo de Proteção Integral as categorias: Estação Ecológica; Reserva Biológica; Parque Nacional; Monumento Natural; e Refúgio de Vida Silvestre. E ao grupo de Uso Sustentável as categorias: Área de Proteção Ambiental; Área de Relevante Interesse Ecológico; Floresta Nacional; Reserva Extrativista; Reserva de Fauna; Reserva de Desenvolvimento Sustentável; e Reserva Particular do Patrimônio Natural (Brasil, 2000).
} 
1998, o governo do estado implementou seu Plano Nacional de Gerenciamento Costeiro - PEGC (São Paulo, 1998), definindo a zona costeira do estado em uma porção terrestre e outra marinha. Em sua área terrestre, inclui os municípios costeiros a partir do divisor de águas de drenagem atlântica (totalizando 36 municípios $^{3}$ ) e, na área marinha, até a isóbata de 23,6 m. Esta definição pode ser considerada adequada, na porção terrestre, por conciliar critérios físicos (bacia de drenagem) e a utilização dos municípios como unidade de gestão (Loitzenbauer \& Mendes, 2014). Para fins de gestão, dividiu-se a zona costeira em quatro setores: Litoral Norte; Baixada Santista; Complexo estuarino-lagunar de Iguape-Cananéia; e Vale do Ribeira.

A região da Baixada Santista se destaca pela presença de uma Região Metropolitana com cerca de 1,7 milhão de habitantes, do porto de Santos e do complexo industrial de Cubatão. O Litoral Norte pela presença de cidades médias-pequenas, fortemente voltadas ao turismo de veraneio e com alguma atividade portuário-industrial na região de São Sebastião e Caraguatatuba. A região do Complexo estuarino-lagunar de Iguape-Cananéia e do Vale do Ribeira se caracteriza por contínuos de Mata Atlântica preservada, associados a pequenas cidades e aglomerados urbanos, predominando atividades pesqueiras, rurais e de turismo. As diferentes atividades socioeconômicas nesses setores geram pressão sobre os ecossistemas naturais, especialmente aquelas associadas ao uso e ocupação desordenado do solo, à especulação imobiliária, a atividades industriais, portuárias e agropecuárias, entre outras.
Em todos os setores existem UCs, tanto terrestres quanto marinhas, sendo a zona costeira paulista historicamente um território no qual esforços vêm sendo aplicados para a conservação ambiental por meio de UCs. A Mata Atlântica, bioma predominante na região, é considerada um hotspot mundial para a conservação da biodiversidade (Myers et al., 2000), motivando a criação de boa parte das UCs. Da mesma forma, abriga diversas comunidades locais que historicamente vêm se utilizando de seus recursos naturais, tanto os terrestres quanto os dos ambientes marinho-costeiros associados (Begossi, 2004). A relação das UCs com as comunidades locais foi marcada por alguns conflitos socioambientais, especialmente com aquelas do grupo de proteção integral (Ferreira, 2004). Com a consolidação do SNUC, outras categorias de UCs foram criadas, possibilitando conciliar a conservação da biodiversidade com a reprodução dos modos de vida das comunidades locais (Machado et al., 2011). Por outro lado, a efetividade destas UCs em atingir seus objetivos depende da implementação de seus instrumentos de gestão, o conselho gestor e o plano de manejo.

Devido à importância da zona costeira paulista para a conservação da biodiversidade e para a manutenção da diversidade sociocultural, o presente estudo teve por objetivo realizar um levantamento das UCs criadas na zona costeira do estado de São Paulo, considerando as diferentes categorias e sua abrangência, a implementação dos instrumentos de gestão e as implicações para a gestão costeira. O foco do estudo são as áreas protegidas enquadra-

\footnotetext{
${ }^{3}$ Litoral norte: Ubatuba, Caraguatatuba, São Sebastião e Ilhabela; Baixada santista: Bertioga, Guarujá, Santos, São Vicente, Cubatão, Praia Grande, Mongaguá, Itanhaém e Peruíbe; Complexo estuarino-lagunar de Iguape-Cananéia: Iguape, Ilha Comprida e Cananeia; e Vale do Ribeira: São Lourenço da Serra, Juquitiba, Pedro de Toledo, Itariri, Miracatu, Tapiraí, Juquiá, Sete Barras, Registro, Pariquera-Açu, Jacupiranga, Cajati, Barra do Turvo, Eldorado, Iporanga, Itaóca, Apiaí, Ribeira, Barra do Chapéu e Itapirapuã Paulista.
} 
das no SNUC, sem considerar outras tipologias de áreas protegidas existentes na zona costeira paulista que ainda necessitam ser reavaliadas conforme o SNUC (e.g., Sá et al., 2017). Espera-se estabelecer um panorama das UCs criadas na zona costeira e marinha do estado de São Paulo, contribuindo para o conhecimento das estratégias de conservação da zona costeira paulista e sua implicação para a gestão costeira.

\section{Metodologia}

Foram levantadas as Unidades de Conservação (UCs) (federais, estaduais e municipais), criadas na zona costeira do estado de São Paulo. A zona costeira foi considerada aquela definida no Plano Estadual de Gerenciamento Costeiro (PEGC) de São Paulo (São Paulo, 1998), abrangendo, na porção terrestre, o território dos 36 municípios costeiros. Na porção marinha, o PEGC inclui a área marinha até a isóbata de 23,6 m, mas, para efeito desse levantamento, foi incluída uma porção mais extensa, considerando a maior abrangência territorial das UCs no ambiente marinho.

Para cada UC foram levantadas as seguintes informações: categoria; data de criação; área total (em ha); localização (por setor); nível administrativo; existência de plano de manejo e ato normativo que o instituiu; existência de outros planos de gestão; e existência de conselho gestor e ato normativo que o instituiu. Os dados foram levantados a partir de consulta a documentos (tais como legislações, trabalhos acadêmicos e relatórios técnicos), sites e bancos de dados governamentais (por exemplo, o Cadastro Nacional de Unidades de Conservação - CNUC - do Ministério do Meio Ambiente). Eventualmente, foram realizados contatos diretos com responsáveis pelas UCs por meio de telefone e e-mail, para verificação e validação de informações. As informações foram compiladas em uma planilha de dados (Microsoft Office Excel) (disponível em Material Suplementar do artigo) e analisadas com o uso de estatísticas descritivas caracterizando o panorama das UCs criadas no litoral do estado 4 .

\section{Resultados e discussão}

\subsection{Categorias de UCs e sua distribuição}

Foi levantado um total de 74 Unidades de Conservação (UCs) na zona costeira de São Paulo, sendo $62 \%$ de uso sustentável e $38 \%$ de proteção integral (Tabela 1). Isto representa, conforme dados do Cadastro Nacional de Unidades de Conservação (CNUC) (MMA, 2017)5 , 32\% do total das UCs do estado de São Paulo. Este percentual é expressivo se considerarmos que a zona costeira corresponde apenas a 8,6\% (IBGE, 2017) do território estadual e concentra cerca de um terço das UCs (Figura 1).

\footnotetext{
${ }^{4}$ No caso de eventuais divergências de informações, foi sempre priorizado o dado disponível nos atos normativos. Em algumas situações, houve dificuldade de se obter o ato normativo, utilizando o dado de sites e bancos de dados governamentais. No caso de falta de clareza da informação, foi realizado contato direto com os responsáveis. As maiores dificuldades do levantamento de informações se deram na esfera municipal, especialmente para identificar as UCs e obter seus atos normativos.

${ }^{5}$ Em consulta realizada em fevereiro de 2018, para o estado de São Paulo estavam registradas 229 UCs. O CNUC do Ministério do Meio Ambiente é atualizado periodicamente. Porém, nem todas as UCs existentes estão cadastradas no CNUC. Por exemplo, Pinto et al. (2017) identificaram que apenas $23 \%$ das UCs municipais de um levantamento por eles realizado estavam cadastradas no CNUC.
} 
TABELA 1 - Número e área das Unidades de Conservação por categoria e grupo na zona costeira do estado de São Paulo.

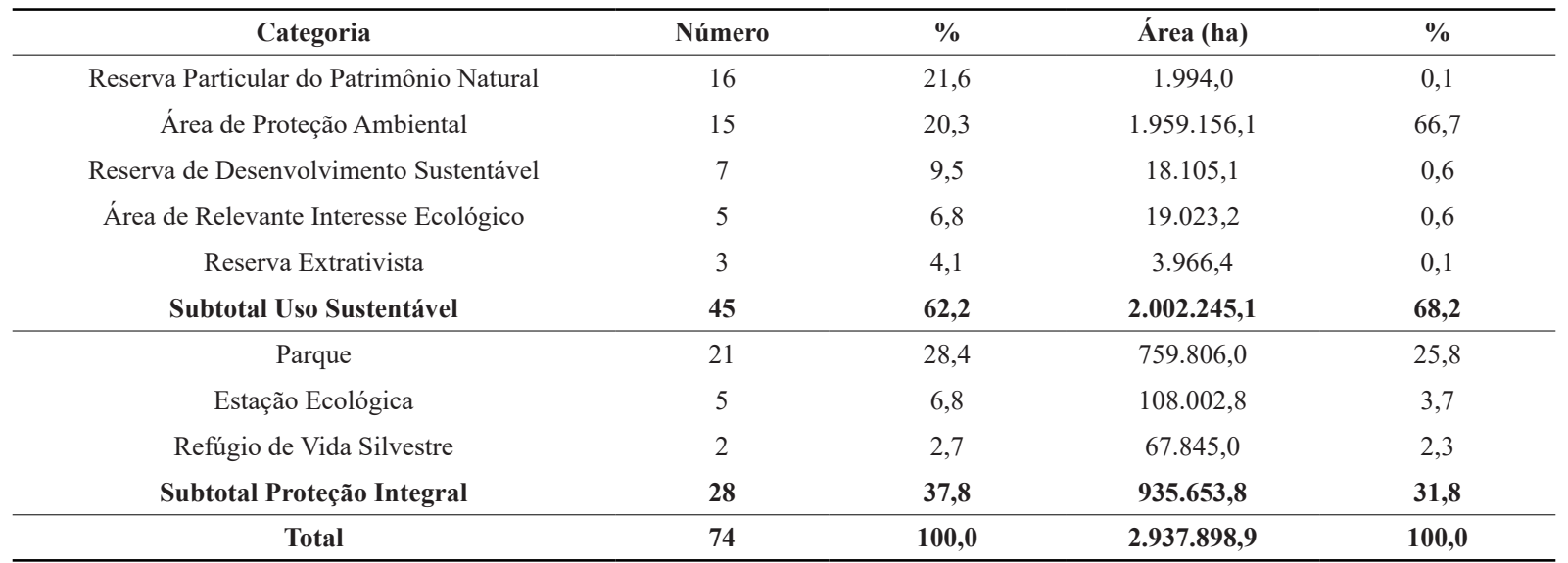

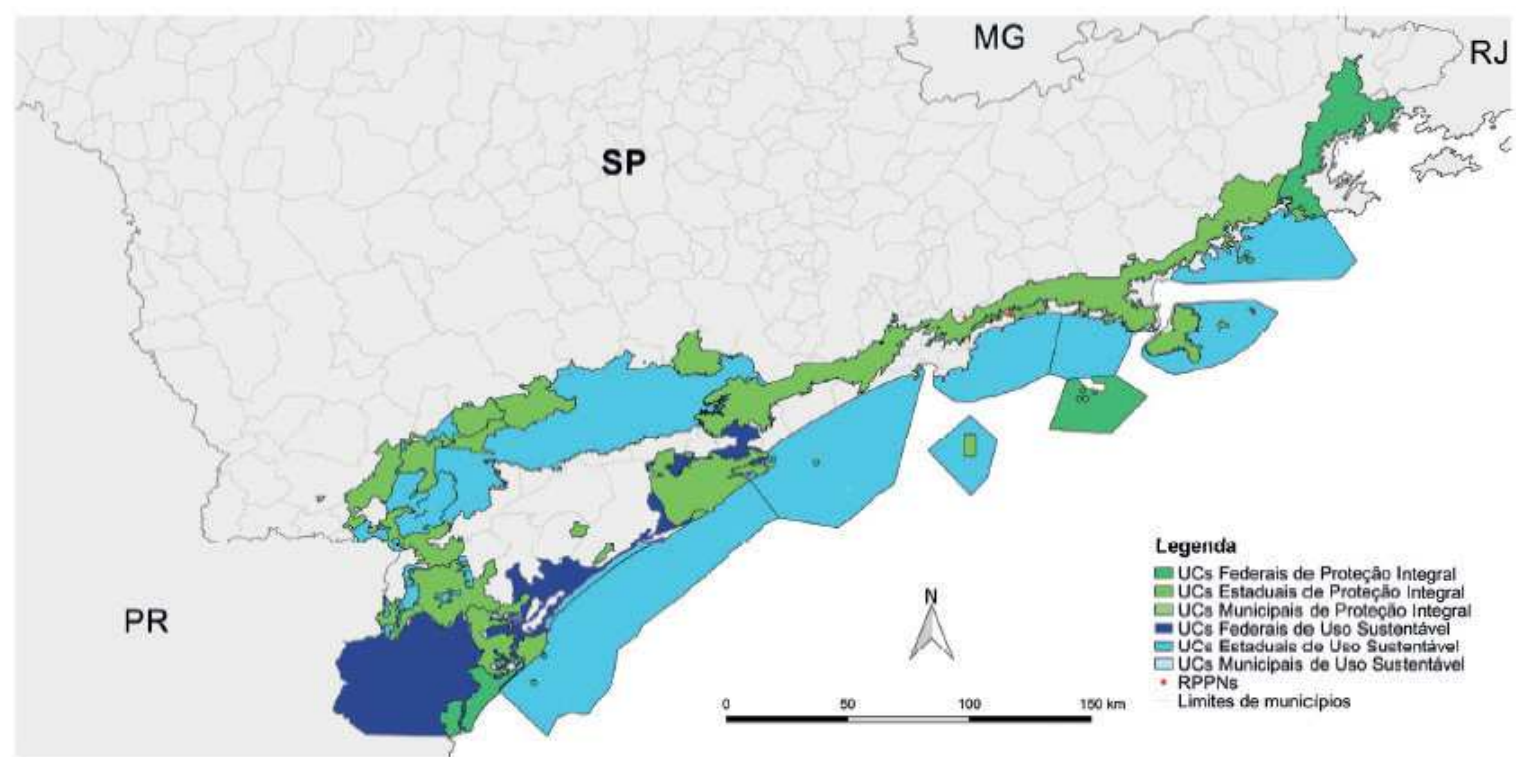

FIGURA 1 - Unidades de Conservação (UCs) de proteção integral e uso sustentável na zona costeira do estado de São Paulo. As seguintes UCs não constam no mapa: UCs estaduais - Área de Relevante Interesse Ecológico da Zona da Vida Silvestre de Ilha Comprida; Estação Ecológica dos Banhados de Iguape; e Parque Estadual Restinga de Bertioga. UCs municipais - Área de Proteção Ambiental Municipal Serra do Guararu; Área de Proteção Ambiental Baleia-Sahy; Área de Proteção Ambiental Alcatrazes; Área de Proteção Ambiental Itaçuce; Parque Municipal Ilha Rio da Praia; Parque Natural Municipal do Juqueriquerê; e Parque Natural Municipal Engenho São Jorge dos Erasmos. PR = Paraná; SP = São Paulo; $\mathrm{MG}=$ Minas Gerais; e RJ = Rio de Janeiro.

FONTE: Elaborado pelos autores com base nos dados de dezembro de 2017 do Cadastro Nacional de Unidades de Conservação (CNUC) e do Sistema Informatizado de Monitoria de RPPN (SIMRPPN), do Instituto Chico Mendes de Conservação da Biodiversidade (ICMBio), Ministério do Meio Ambiente (MMA). 
Com relação à distribuição pelos diferentes setores da zona costeira paulista, todos contam com um número similar de UCs de proteção integral e o litoral norte com um menor número de UCs de uso sustentável. Em termos de área, o Complexo Estuarino-Lagunar de Iguape e Cananéia conta com uma área mais expressiva de UCs de uso sustentável com relação às de proteção integral (Tabela 2). Isto se explica pela presença de duas grandes APAs na região, abrangendo parte significativa do território terrestre (Área de Proteção Ambiental Cananéia-Iguape-Peruíbe) e marinho (Área de Proteção Ambiental Marinha Litoral Sul), ao mesmo tempo em que a maior UC de proteção integral da zona costeira paulista (Parque Estadual da Serra do Mar) não abrange este setor do litoral. Em termos absolutos, o Vale do Ribeira abrange a maior área com UCs e o CELIC, a menor.

Dentre as categorias, predomina Parques (P) (28,4\%), seguida por Reserva Particular do Patrimônio Natural (RPPN) (21,6\%), Área de Proteção Ambiental (APA) (20,3\%), Reserva de Desenvolvimento Sustentável (RDS) (9,5\%), Estação Ecológica (ESEC) e Área de Relevante Interesse Ecológico
(ARIE) (ambas com 6,8\%), Reserva Extrativista (RESEX) (4,1\%) e Refúgio da Vida Silvestre (RVS) (2,7\%). Não há nenhuma UC na categoria Reserva Biológica, Monumento Natural, Floresta Nacional e Reserva de Fauna (Tabela 1).

Apesar de a categoria Parque ser a predominante, as APAs abrangem mais que o dobro em termos de área (ha), o que explicaria a maior abrangência das UCs de uso sustentável (2.002.245,1 ha, 68,2\%) em relação às de proteção integral (935.653,8 ha, 31,8\%). As demais categorias representam um total de área ocupada bastante inferior aos Parques e APAs. No caso das RPPNs, apesar do número relativamente alto em relação às demais categorias, a área ocupada é a menor. Esta categoria, normalmente relacionada à propriedade privada individual (Pellin \& Ranieri, 2016), apresenta área reduzida, em sua maioria até 500 ha (Pellin, 2010) (média de 124,6 ha no presente estudo, variando de 0,7 a 709,6 ha).

A primeira UC criada na zona costeira de São Paulo foi em 1958 (Parque Estadual Turístico do Alto Ribeira) e até o início dos anos de 1980 os Parques, quase todos estaduais (à exceção do Parque

TABELA 2 - Número e área das Unidades de Conservação por grupo nos diferentes setores da zona costeira do estado de São Paulo. VL= Vale do Ribeira; CELIC = Complexo estuarino-lagunar de Iguape e Cananéia; BS = Baixada Santista; e LN = Litoral Norte.

\begin{tabular}{|c|c|c|c|c|c|c|}
\hline \multirow{2}{*}{ Setor } & \multicolumn{2}{|c|}{ Proteção Integral } & \multicolumn{2}{|c|}{ Uso Sustentável } & \multicolumn{2}{|c|}{ Total } \\
\hline & N (\%) & Área (\%) & N (\%) & Área (\%) & $\mathbf{N}$ & Área \\
\hline VL & $\begin{array}{c}9 \\
(39 \%)\end{array}$ & $\begin{array}{c}635.812,9 \\
(47 \%)\end{array}$ & $\begin{array}{c}14 \\
(61 \%)\end{array}$ & $\begin{array}{c}711.755,4 \\
(53 \%)\end{array}$ & 23 & $1.347 .568,3$ \\
\hline CELIC & $\begin{array}{c}9 \\
(41 \%)\end{array}$ & $\begin{array}{c}240.462,9 \\
(28 \%)\end{array}$ & $\begin{array}{c}13 \\
(59 \%)\end{array}$ & $\begin{array}{c}617.728,8 \\
(72 \%)\end{array}$ & 22 & $858.191,7$ \\
\hline $\mathrm{LN}$ & $\begin{array}{c}7 \\
(44 \%)\end{array}$ & $\begin{array}{c}516.780,9 \\
(55 \%)\end{array}$ & $\begin{array}{c}9 \\
(56 \%)\end{array}$ & $\begin{array}{c}417.405,8 \\
(45 \%)\end{array}$ & 16 & $934.186,7$ \\
\hline
\end{tabular}


Nacional da Serra da Bocaina), compreendiam a totalidade de áreas protegidas na zona costeira (Figura 2). Em sua maioria, abrangia ilhas e porções da Serra do Mar. Os Códigos Florestais de 1934 e de 1965 (Brasil, 1934; 1965) eram os instrumentos legais que consolidavam à época a criação destas primeiras áreas protegidas (Medeiros, 2006).

Entre os anos 1980 até o final dos anos 1990, outras categorias foram criadas, além dos Parques, como as Áreas de Proteção Ambiental, Áreas de Relevante Interesse Ecológico, Estações Ecológicas e Reservas Particulares do Patrimônio Natural, com base em outros instrumentos legais que instituíram novas categorias de áreas protegidas (Brasil, 1981;
1984; 1996). Além de ilhas e da Serra do Mar, estas novas áreas abrangiam porções da planície costeira e do Vale do Ribeira sob domínio da Mata Atlântica, bem como as primeiras áreas protegidas em ambientes estuarinos e marinhos (Área de Proteção Ambiental Cananéia-Iguape-Peruíbe, Estação Ecológica Tupinambás, Estação Ecológica dos Tupiniquins, Parque Estadual Marinho da Laje de Santos e Parque Estadual Xixová-Japuí). A partir dos anos 2000, com a consolidação do SNUC, diversas outras UCs foram criadas, acrescentando as categorias Refúgio da Vida Silvestre, Reserva de Desenvolvimento Sustentável e Reserva Extrativista.

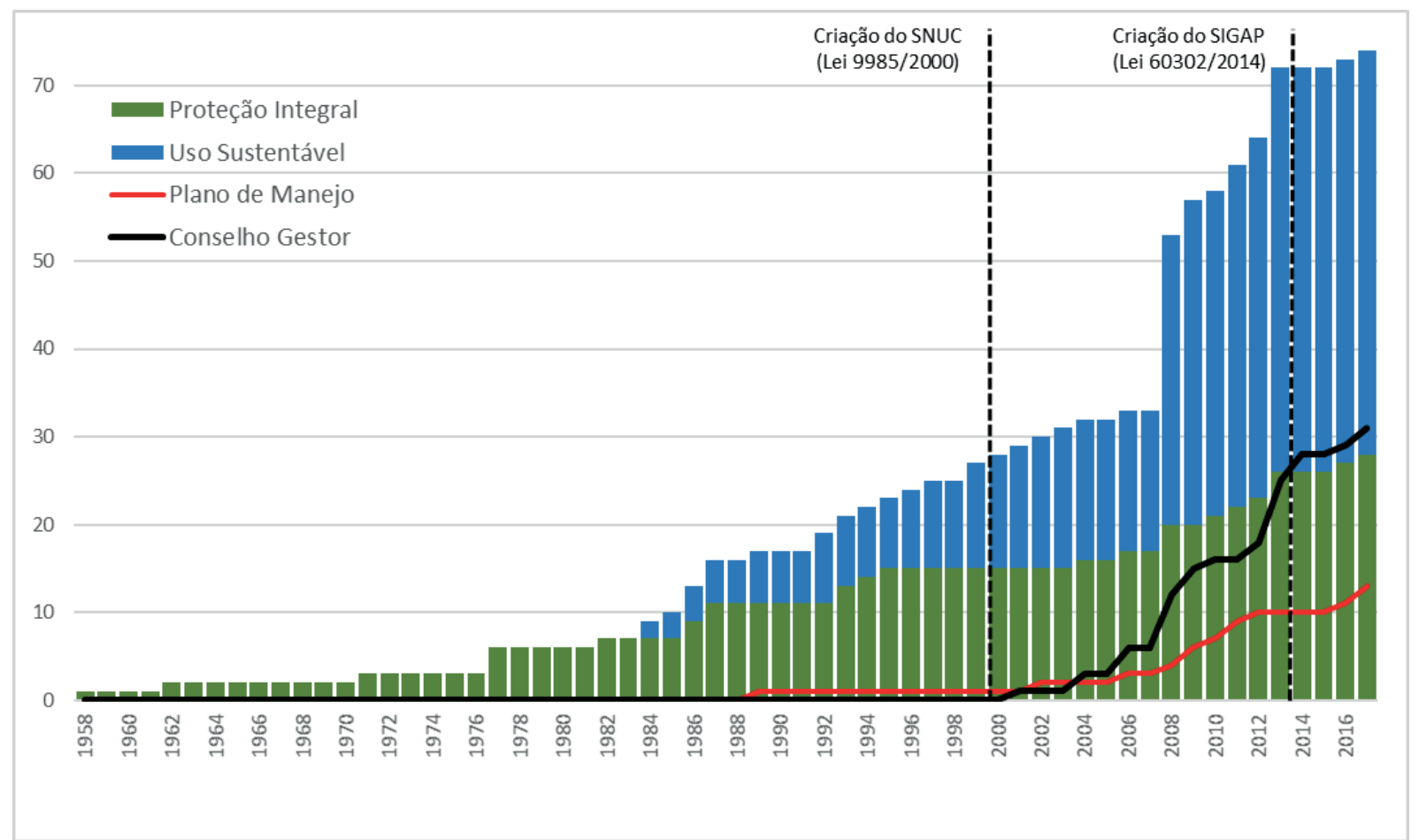

FIGURA 2 - Evolução do número de Unidades de Conservação, planos de manejo e conselhos gestores implementados na zona costeira de São Paulo entre 1958 e 2017. SNUC = Sistema Nacional de Unidades de Conservação; SIGAP = Sistema de Informação e Gestão de Áreas Protegidas e de Interesse Ambiental do Estado de São Paulo. 
As UCs de uso sustentável surgem principalmente a partir dos anos 2000, após a instituição da Lei do SNUC, predominando na zona costeira paulista em relação às UCs de proteção integral a partir de 2008 (Figura 2). Entre 2008 e 2013, há um considerável aumento de UCs na zona costeira, principalmente devido a três motivos. Primeiro, a transformação do antigo Parque Estadual de Jacupiranga em Mosaico de Unidades de Conservação do Jacupiranga (São Paulo, 2008a), dando origem a novas UCs, especialmente APA, RDS e RESEX. Segundo, a criação das APAs marinhas (litoral norte, centro e sul) e duas ARIEs, compondo, juntamente com outras UCs já existentes, o Mosaico das Ilhas e Áreas Marinhas Protegidas do Litoral Paulista (São Paulo, 2008b). E terceiro, a alteração dos limites da Estação Ecológica da Juréia-Itatins, dando origem a dois Parques, duas RDS e uma REVIS, constituindo o Mosaico de Unidades de Conservação da Juréia-Itatins (São Paulo, 2013). Nesse período, há ainda a criação de dez RPPNs, nove no âmbito estadual, possivelmente em decorrência da institucionalização do Programa Estadual de Apoio às Reservas Particulares do Patrimônio Natural em 2006 (São Paulo, 2006).

A grande maioria das UCs (69\%) foi criada na esfera estadual. No ano de 1986 foram criadas a Secretaria de Meio Ambiente do Estado de São Paulo (SMA) e a Fundação para a Conservação e a Produção Florestal do Estado de São Paulo (Fundação Florestal), esta última vinculada à SMA e atuando conjuntamente com o Instituto Florestal, sendo responsável pela gestão de grande parte das UCs estaduais (São Paulo, 2006; 2009), o que potencialmente fortaleceu o processo de criação de UCs estaduais a partir desse período.

A partir de 2014, o estado estabeleceu diretrizes próprias com relação à criação e à implementação de UCs estaduais por meio do Sistema de Informação e Gestão de Áreas Protegidas e de Interesse Ambiental do Estado de São Paulo - SIGAP (São Paulo, 2014). Apesar de boa parte das UCs estaduais ser anterior à política, o SIGAP consolidou as normas referentes tanto à criação quanto à implementação das UCs e de seus instrumentos em nível estadual. Porém, ainda não é possível avaliar em que medida esta política de fato vem resultando em maiores esforços para criar e implementar a gestão das UCs estaduais.

Destaca-se ainda, na zona costeira de São Paulo, a criação de Mosaicos de Unidades de Conservação entre 2006 e 2013, totalizando seis dos sete mosaicos do estado, quatro deles criados na esfera estadual. Com relação ao panorama nacional, os mosaicos existentes no litoral de São Paulo representam $25 \%$ dos mosaicos brasileiros, segundo levantamento de Pellin et al. (2017). Os Mosaicos ${ }^{6}$ são uma estratégia de gestão integrada territorial, visando definir objetivos para o território do Mosaico que compartilha uma identidade comum, por meio de uma articulação interinstitucional que operacionalize as ações de gestão (Pinheiro, 2010; Pellin et al., 2017). Nesse contexto, sua presença no litoral paulista é bastante significativa, uma vez que 44 das UCs costeiras e marinhas (59\% do total) fazem parte de ao menos um Mosaico. Por outro

${ }^{6}$ O Art. 26 da Lei do SNUC estabelece: “Quando existir um conjunto de unidades de conservação de categorias diferentes ou não, próximas, justapostas ou sobrepostas, e outras áreas protegidas públicas ou privadas, constituindo um mosaico, a gestão do conjunto deverá ser feita de forma integrada e participativa, considerando-se os seus distintos objetivos de conservação, de forma a compatibilizar a presença da biodiversidade, a valorização da sociodiversidade e o desenvolvimento sustentável no contexto regional” (Brasil, 2000). 
lado, poucos implementaram seus instrumentos de gestão previstos, tais como conselho gestor (apenas Mosaicos da Bocaina e Lagamar), plano de manejo (nenhum deles), planejamento estratégico, secretaria executiva e projetos específicos (presentes apenas no Mosaico da Bocaina) (Pellin et al., 2017).

Já as UCs municipais representam apenas $12 \%$ das UCs da zona costeira, sendo cinco APAs e quatro Parques. Um dos incentivos que vem promovendo a criação de UCs municipais no Brasil está relacionado ao recebimento de parcela do Imposto sobre Circulação de Mercadorias e Serviços (ICMS) com base em critérios ambientais, incluindo a existência de UCs em seu território (Euclydes \& Magalhães, 2006; Conti et al., 2015; Pinto et al., 2015; Pinto et al., 2017). São Paulo adota uma política de ICMS Ecológico desde 1993 (São Paulo, 1993), mas o repasse está vinculado aos espaços territoriais especialmente protegidos sob gestão estadual (SMA, 2015), o que possivelmente não gera um incentivo para a criação de UCs na esfera municipal, como ocorre em outros estados.

\subsection{Implementação dos instrumentos de gestão}

A implementação dos principais instrumentos de gestão, conselho gestor e plano de manejo, se deu principalmente a partir de 2008 . No caso dos conselhos, sua obrigatoriedade só foi instituída a partir do SNUC, em 2000. Os planos de manejo apresentaram um ritmo mais lento de criação ao longo do tempo em relação aos conselhos (Figura 2).

Cerca de metade (53\%) das UCs já instituiu conselho, enquanto que apenas $19 \%$, o plano de manejo. As UCs de uso sustentável e de proteção in- tegral avançaram de maneira similar na implementação dos conselhos (53\% e 54\%, respectivamente), porém, no caso do plano de manejo, as de proteção integral avançaram mais (43\%) do que as de uso sustentável (4\%). Dentre as categorias de UCs, as RESEX são as com maior percentual de conselho instituído (100\%) e as RDS com o menor (14\%). No caso do plano de manejo, RVS apresenta o maior percentual de implementação (50\%), enquanto que todas as demais categorias do grupo de uso sustentável ainda não implementaram esse instrumento, à exceção de uma RESEX (Mandira) e uma APA (APA Municipal Serra do Guararu) (Tabela 3). No que diz respeito à esfera administrativa, as UCs federais têm sido mais eficientes na implementação dos conselhos (88\% das UCs com conselho) em relação às estaduais (54\%) e às municipais $(22 \%)$, o mesmo ocorrendo para os planos de manejo: federais (36\%), estaduais (16\%) e municipais (11\%).

Considerando que apenas a partir do SNUC, em 2000, tornou-se obrigatória a criação de conselhos para as UCs, desde então o tempo médio de criação desse instrumento na zona costeira paulista foi de 5,2 anos, variando de 0 até 14 anos. Apesar da relevância desse instrumento para a efetividade da gestão, incluindo sua participação ativa no desenvolvimento e na implementação do plano de manejo, tanto a lei federal quanto a estadual não determinam um prazo em que os conselhos devam ser implementados. No caso dos planos de manejo, o tempo médio de criação foi de 21,6 anos, variando de 1 a 50 anos. A Lei do SNUC determina que este instrumento seja implementado no prazo de cinco anos a partir da data de sua criação e, no caso das UCs estaduais, o SIGAP é mais rigoroso, determinando um prazo de até três anos. Apenas dois (REVIS do Arquipélago de Alcatrazes e APA 
TABELA 3 - Número e percentual de conselho gestor e plano de manejo instituídos nas Unidades de Conservação da zona costeira do estado de São Paulo.

\begin{tabular}{|c|c|c|}
\hline Categoria & $\begin{array}{c}\text { Conselho Gestor instituído - núme- } \\
\text { ro }(\%)\end{array}$ & $\begin{array}{c}\text { Plano de Manejo instituído - } \\
\text { número }(\%)\end{array}$ \\
\hline Reserva Particular do Patrimônio Natural & $-{ }^{a}$ & $0(0 \%)$ \\
\hline Reserva de Desenvolvimento Sustentável & $1(14 \%)$ & $0(0 \%)$ \\
\hline Área de Relevante Interesse Ecológico & $3(60 \%)$ & $0(0 \%)$ \\
\hline Subtotal Uso Sustentável & $16(53 \%)$ & $2(4 \%)$ \\
\hline Parque & $11(52 \%)$ & $9(43 \%)$ \\
\hline Estação Ecológica & $3(60 \%)$ & $2(40 \%)$ \\
\hline Refúgio de Vida Silvestre & $1(50 \%)$ & $1(50 \%)$ \\
\hline
\end{tabular}

Municipal Serra do Guararu) dos 19 planos de manejo implementados cumpriram o prazo de cinco anos determinado no SNUC.

Algumas UCs que não possuem plano de manejo contam com outros tipos de planos de gestão específicos ou provisórios. É o caso dos Parques Estaduais da Laje de Santos, da Restinga de Bertioga e do Itinguçu, que possuem plano emergencial de uso, da Reserva de Desenvolvimento Sustentável de Lavras, que possui um plano de utilização, e dos Parques Estaduais Caverna do Diabo, do Rio Turvo, Turístico do Alto Ribeira (PETAR) e Intervales, que possuem plano de manejo espeleológico. Estes instrumentos de gestão estão previstos no SIGAP e têm caráter provisório, até que se estabeleça o plano de manejo, com exceção do plano de manejo espeleológico, previsto para a gestão de cavidade natural subterrânea (CONAMA, 2004).

\subsection{Implicações para a gestão costeira}

A reconhecida relevância das zonas costeiras dos pontos de vista biofísico, socioeconômico e cultural (Cicin-Sain \& Knecht, 1998) tem levado a esforços de conservação, incluindo a criação de áreas protegidas. No âmbito nacional, existe a preocupação em atingir as metas internacionais de proteção dos ecossistemas marinhos e costeiros, as quais, em certa medida, vêm sendo alcançadas no que diz respeito aos ecossistemas costeiros, mas ainda muito distantes para os ambientes marinhos (MMA, 2010). Por outro lado, considerando que boa parte dos ecossistemas abrangidos por UCs está em categorias de uso sustentável, especialmente em APA, alguns autores contestam a efetividade dessas UCs em conservar a integridade dos ecossistemas costeiros. Isto porque, no caso das APAs terrestres,

aA legislação federal (Brasil, 2000; 2002; 2006) não menciona a necessidade de conselho gestor para esta categoria e a legislação estadual (São Paulo, 2014), em seu artigo 22, determina que a RPPN não contará com conselho gestor. 
devido a sua grande extensão e ao predomínio de terras privadas, a simples existência do diploma legal de criação não garante sua efetividade; esta depende da implementação dos instrumentos de gestão para que a UC alcance seus objetivos de conservação (Oliveira, 2002; Artaza-Barrios \& Schiavetti, 2007; MMA, 2010; Pádua, 2011).

No contexto da zona costeira de São Paulo, as UCs apresentam especial relevância, uma vez que cobrem uma porção significativa do território, incluindo quase todo o Mar Territorial (Figura 1). Isto implica que sejam um dos principais instrumentos para a gestão e a governança do uso dos espaços e dos recursos costeiros. Por exemplo, na porção marinha da zona costeira, o conjunto de UCs que compõem o Mosaico das Ilhas e Áreas Marinhas Protegidas do Litoral Paulista abrange uma área expressiva ao longo de todo o litoral do estado. Mesmo que boa parte do Mosaico seja composta por APAs, a natureza pública do ambiente marinho gera oportunidades de gestão diferentes daquelas do ambiente terrestre (Prates \& Blanc, 2007; MMA, 2010), o que potencialmente minimiza algumas das ressalvas feitas às APAs terrestres.

Além dos esforços de criação das UCs, para que as mesmas sejam consideradas efetivas, há a necessidade de ampliar a consolidação de seus instrumentos de gestão. Os conselhos gestores, apesar de sua institucionalização ter avançado a partir de 2008, ainda não abrangem uma parcela importante das UCs criadas. A efetividade da gestão depende da implementação do conselho e de seu funcionamento ativo, oportunizando a participação social (ICMBio/WWF, 2011; Abirached et al., 2014; Banzato, 2014; Oliveira-Júnior et al., 2016). Um dos avanços reconhecido sobre o SNUC está em valorizar o envolvimento e a participação de diferentes atores no processo de gestão, em especial daquelas comunidades locais diretamente afetadas pelas UCs (Vivacqua \& Vieira, 2005; Peccatiello, 2011). Esta participação, também endossada pela política estadual de áreas protegidas de São Paulo (SIGAP), apresenta avanços no envolvimento das comunidades locais relacionadas às UCs costeiras paulistas, mas persistem ainda problemas de representatividade, de diferenças de poder entre os atores nos arranjos de governança, das distintas visões entre gestores e comunidades, e da capacidade dos conselhos em de fato atender determinadas demandas locais (Bockstael et al., 2016; Calvimontes \& Ferreira, 2016; Assis, 2017; Rodrigues \& Magro, 2017).

O contexto histórico da criação de UCs na zona costeira de São Paulo é marcado por conflitos socioambientais fundiários e de uso dos recursos naturais. Antonio Carlos Diegues, em 2001, afirmava que em 37,5\% dos Parques do estado de São Paulo havia ocupação humana, seja ela por populações tradicionais ou não (Diegues, 2001). Alguns desses conflitos incluem o caso da Estação Ecológica da Juréia-Itatins, do Parque Estadual da Ilha do Cardoso, do Parque Estadual da Serra do Mar, dentre outros (Diegues, 2001; Sanches, 2001; Ferreira, 2004; Piedade \& Molina, 2013; Calvimontes \& Ferreira, 2016). Parte desses conflitos levou a processos de recategorização e alteração de limites de UCs de proteção integral, notadamente no caso da criação do Mosaico de Unidades de Conservação do Jacupiranga e da Juréia-Itatins. Nas últimas décadas, a organização política das comunidades (Ferreira, 2004), associada à construção de instrumentos legais de reconhecimento do direito de certas populações que habitam UCs de proteção integral (Piedade \& Molina, 2013), tem gerado diferentes 
situações de tratamento dos conflitos fundiários e de uso dos recursos. Porém, conflitos ainda persistem (Calvimontes \& Ferreira, 2016).

Neste sentido, a implementação dos conselhos é um passo importante para lidar de forma participativa com as situações de conflito e também para a elaboração de estratégias de manejo dos recursos naturais. Esses espaços de gestão possibilitam uma perspectiva de gestão compartilhada (ou cogestão) (Kalikoski et al., 2009), com graus diferenciados de direitos entre os atores na tomada de decisão conforme a natureza do conselho, predominando os consultivos em relação aos deliberativos (este último somente nas RDS e RESEX). A consolidação dos conselhos também é fundamental para dar legitimidade ao processo de elaboração ou revisão dos planos de manejo (Vivacqua \& Vieira, 2005; Rainho, 2014; Bockstael et al., 2016), evitando que as UCs acabem se tornando mais uma fonte de pressão sobre os modos de vida das comunidades locais (Hanazaki et al., 2013; Alves \& Hanazaki, 2015; Faraco et al., 2016).

O que observamos na zona costeira de São Paulo é um percentual intermediário de UCs com conselhos gestores instituídos, mas muito inferior no caso do plano de manejo, sendo este último praticamente inexistente nas UCs de uso sustentável. Considerando que estas UCs possibilitam o uso direto, os planos de manejo, construídos de forma participativa, são fundamentais para estabelecer o uso adequado dos recursos naturais. A ausência desses instrumentos implica não somente ao que convencionalmente se denominou de paper parks (isto é, na ausência de recursos e estratégias claras de gestão as áreas protegidas acabam apenas existindo legalmente, mas sem atingir seus objetivos para as quais foram criadas), mas também em limitar as possibilidades em lidar com os conflitos socioambientais historicamente estabelecidos com a criação da UC.

Alguns problemas estruturais de governança, incluindo falta de financiamento, baixa coordenação interinstitucional, gestão deficiente das UCs, entre outros, são fatores apontados que enfraquecem o SNUC e sua implementação na esfera federal (Gerhardinger et al., 2011). Internacionalmente, a falta de uma equipe de gestão adequada, bem como de recursos financeiros, vêm sendo apontadas como fatores cruciais para a efetividade de áreas marinhas protegidas (Gill et al., 2017). No caso da zona costeira paulista, onde $68 \%$ das UCs estão sob gestão estadual, alguns autores também apontam problemas na gestão estadual, incluindo dotação orçamentária insuficiente, baixos salários e falta de planos de carreira de funcionários, falta de capacitação técnica, precarização dos serviços prestados e excesso de cargos comissionados, por vezes sem a formação adequada (Faria, 2004; Reis \& Queiroz, 2017). Nesse contexto, a superação dessas deficiências estruturais por vezes depende do protagonismo individual de determinados gestores para alcançar a capacidade de governança necessária, uma vez que o sistema não possibilita a estrutura de trabalho necessária (Gerhardinger $e t$ al., 2011; Macedo et al., 2013). Pode-se acrescentar ainda outros entraves de caráter político-institucional associados à gestão costeira, como pressões econômicas que tensionam as políticas públicas e as descontinuidades administrativas, com alterações do quadro técnico e das prioridades institucionais (Polette et al., 2004). 
As metas internacionais de conservação ${ }^{7}$ são compromissos prioritários para a conservação da biodiversidade e para a manutenção de inúmeros serviços ecossistêmicos a ela associados, mobilizando esforços para a criação de UCs no Brasil (MMA, 2010). A busca pela equidade no planejamento e na gestão dessas áreas protegidas é um dos desafios postos, especialmente por conta da assimetria de poder ente os atores envolvidos (Hill et al., 2016).

As UCs na zona costeira de São Paulo possibilitam hoje a conservação dos ecossistemas remanescentes da região, frente às pressões pela urbanização associada às atividades urbano-industriais e pelo turismo, além de outras atividades produtivas de origem rural. Potencialmente, são também uma oportunidade para a manutenção da diversidade cultural, principalmente das populações tradicionais de origem Caiçara, desde que o planejamento e a implementação das UCs envolvam, desde as fases iniciais, a participação ativa das comunidades, garantindo a equidade nos benefícios gerados pela conservação (Chuenpagdee et al., 2013; Trimble et al., 2014; Bockstael et al., 2016). Considerando o aumento de UCs na zona costeira de São Paulo na última década, estes aspectos são centrais para que as mesmas possam alcançar seus objetivos de conservação ambiental e socioeconômicos, especialmente pelo predomínio das UCs de uso sustentável.

A gestão integrada das UCs com as dinâmicas territoriais da zona costeira pode ser oportunizada pela institucionalização dos Mosaicos de UCs, que vêm sendo criados de maneira bastante expressiva na zona costeira paulista com relação a outras regiões no Brasil. Este processo também depende da consolidação dos instrumentos previstos para a gestão dos Mosaicos, o que ainda não ocorreu.

Outra estratégia de integração inclui a relação com políticas de gerenciamento costeiro. As UCs são afetadas por atividades que têm origem muito além de seus limites, sendo observadas diversas pressões de ocupação sobre as UCs costeiras paulistas (Mello et al., 2011; Inouye et al., 2015). Mecanismos claros de integração com outras políticas podem contribuir para reduzir estas pressões. Isto envolve garantir condições territoriais que possibilitem a conectividade entre as áreas protegidas, a geração e a difusão de informações por meio de programas de monitoramento, o fomento de atividades socioeconômicos regionais condizentes com a manutenção da qualidade ambiental, a integração interinstitucional entre diferentes esferas administrativas e parcerias para capacitação técnica e financiamento (Cicin-Sain \& Belfiore, 2005). Neste aspecto, São Paulo se destaca, entre outros estados, na implementação do seu Plano Estadual de Gerenciamento Costeiro e dos seus instrumentos previstos (Scherer et al., 2010). O Zoneamento Ecológico-Econômico Costeiro (ZEEC), já presente nos setores do litoral norte e da baixada santista, prevê a ocorrência das UCs no território, tratando as mesmas como uma zona especial (Z1AEP - Áreas Especialmente Protegidas). O fortalecimento dos planos de gestão se torna um passo importante tanto para implementar as metas para o ZEEC quanto para alcançar as condições para a integração das UCs com o território e, assim, reduzir as pressões

\footnotetext{
${ }^{7}$ Com relação às zonas costeiras e marinhas, a $10^{a}$ Conferência (COP 10) dos Países Signatários da Convenção de Diversidade Biológica (CDB), realizada em Nagoya, Japão, em outubro de 2010, estabeleceu em sua meta 11 que pelo menos 10\% dessas áreas estejam conservadas por meio do estabelecimento de Áreas Protegidas.
} 
e fomentar as oportunidades que deem suporte aos objetivos das UCs, buscando a qualidade de vida na zona costeira paulista.

\section{Conclusões}

A zona costeira paulista concentra grande parte das UCs do estado, predominando na última década as UCs de uso sustentável com relação às de proteção integral. A presença dessas áreas protegidas e a sua distribuição pelos setores do litoral paulista são essenciais para a preservação dos ambientes costeiros e marinhos, sendo o ambiente marinho coberto quase totalmente por UC. A ação política de criação dessas áreas protegidas é predominantemente estadual e desde 2014 o estado conta com uma política específica para a criação e a implementação das UCs. O histórico de criação foi marcado pela sobreposição de UCs de proteção integral com áreas habitadas, principalmente por populações tradicionais. A recategorização de algumas UCs e sua integração em Mosaicos procurou lidar com alguns desses conflitos, mas que ainda se fazem presentes.

A baixa implementação dos instrumentos de gestão, especialmente os planos de manejo, acaba por não oportunizar estratégias de gestão para lidar com os conflitos e para estabelecer, de forma participativa, o manejo dos recursos naturais. Considerando que a média de criação dos dois principais instrumentos de gestão varia de 5,3 anos para os conselhos e de 21,6 anos para os planos de manejo, se faz clara a necessidade de maiores esforços para a instituição de ambos os instrumentos.

O panorama das UCs criadas na zona costeira e marinha do estado de São Paulo revelou sua importância enquanto instrumento de gestão costeira.
Sua expressiva presença ao longo de todos os setores ressalta a necessidade de uma perspectiva de gestão integrada do território, buscando a equidade dos benefícios gerados por elas, por meio do envolvimento de todos os atores desde o planejamento até a sua gestão.

A criação de Mosaicos vem sendo promovida pela política estadual como estratégia de gestão integrada, resultando hoje na maior concentração de Mosaicos do território brasileiro, os quais abrangem cerca de $60 \%$ das UCs da zona costeira paulista, mas ainda pendente da efetiva implementação por meio de seus instrumentos de gestão. A integração com outras políticas de gestão costeira, em especial o PEGC e seus instrumentos, como o ZEEC, representa outra oportunidade de integração das UCs com as dinâmicas territoriais da zona costeira paulista.

\section{Referências}

Abirached, C. F. de A.; Mendonça, F. C.; Luz, L.; Talbot, V.; Lasmar, V. Conselhos Gestores de Unidades de Conservação federais. Brasília: Instituto Chico Mendes de Conservação da Biodiversidade, Coordenação de Gestão Participativa, 2014. 76 p.

Alves, R. P.; Hanazaki, N. Áreas protegidas marinho-costeiras de Santa Catarina sob a perspectiva das populações locais: contribuições da literatura. Ambiente \& Sociedade, 18(4), 97-118, 2015.

Artaza-Barrios, O. H.; Schiavetti, A. Análise da efetividade do manejo de duas Áreas de Proteção Ambiental do Litoral Sul da Bahia. Revista da Gestão Costeira Integrada, 7(2), 117-128, 2007.

Assis, L. C. A. R. Gestão participativa da Área de Proteção Ambiental Marinha do Litoral Centro do Estado de São Paulo. In: Moura, G. G. M. (Org.). Avanços em oceanografia humana: o socioambientalismo nas Ciências do Mar. Jundiaí, SP: Paco, 2017, p. 159-195. 
Banzato, B. de M. Análise da efetividade das Unidades de Conservação Marinhas de Proteção Integral do estado de São Paulo. São Paulo, Dissertação (Mestrado em Ciência Ambiental) - USP, 2014.

Begossi, A (Org.). Ecologia de pescadores da Mata Atlântica e da Amazônia. São Paulo: Hucitec, Nepam/Unicamp, Nupaub/USP, Fapesp, 2004. 332 p.

Bockstael, E.; Bahia, N. C. F.; Seixas, C. S.; Berkes, F. Participation in protected area management planning in coastal Brazil. Environmental Science and Policy, 60, 1-10, 2016. doi: 10.1016/j.envsci.2016.02.014

Brasil. Decreto $n^{\circ} 23.793$, de 23 de janeiro de 1934. Aprova o Código Florestal. Rio de Janeiro: Presidência da República, Casa Civil, Subchefia para Assuntos Jurídicos, 1934.

Brasil. Lei $n^{\circ} 4.771$, de 15 de setembro de 1965. Institui o novo Código Florestal. Brasília: Presidência da República, Casa Civil, Subchefia para Assuntos Jurídicos, 1965.

Brasil. Lei $n^{\circ}$ 6.902, de 27 de abril de 1981. Dispõe sobre a criação de Estações Ecológicas, Áreas de Proteção Ambiental e dá outras providências. Brasília: Câmara dos Deputados, Centro de Documentação e Informação, 1981.

Brasil. Decreto $n^{\circ} 89.336$, de 31 de janeiro de 1984. Dispõe sobre as Reservas Econômicas e Áreas de Relevante Interesse Ecológico, e dá outras providências. Brasília: Presidência da República, Casa Civil, Subchefia para Assuntos Jurídicos, 1984.

Brasil. Decreto $n^{\circ} 1.922$, de 5 de junho de 1996. Dispõe sobre o reconhecimento das Reservas Particulares do Patrimônio Natural, e dá outras providências. Brasília: Presidência da República, Subchefia para Assuntos Jurídicos, 1996.

Brasil. Lei $n^{\circ}$ 9.985, de 18 de julho de 2000. Regulamenta o art. 225, § $1^{\circ}$, incisos I, II, III e VII da Constituição Federal, institui o Sistema Nacional de Unidades de Conservação da Natureza e dá outras providências. Brasília: Presidência da República, Subchefia para Assuntos Jurídicos, 2000.

Brasil. Decreto $n^{\circ} 4.340$, de 22 de agosto de 2002. Regulamenta artigos da Lei ${ }^{\circ}$ 9.985, de 18 de julho de 2000, que dispõe sobre o Sistema Nacional de Unidades de Conservação da Natureza - SNUC, e dá outras providências. Brasília: Presidência da República, Subchefia para Assuntos
Jurídicos, 2002.

Brasil. Decreto $n^{\circ} 5.746$, de 5 de abril de 2006. Regulamenta o art. 21 da Lei n ${ }^{\circ} 9.985$, de 18 de julho de 2000 , que dispõe sobre o Sistema Nacional de Unidades de Conservação da Natureza. Brasília: Presidência da República, Subchefia para Assuntos Jurídicos, 2006.

Calvimontes, J.; Ferreira, L. da C. Bandidos na Serra do Mar: conflitos, estratégias e usos múltiplos dos recursos naturais na Mata Atlântica, São Paulo. Desenvolvimento e Meio Ambiente, 38, 77-99, 2016. doi: 10.5380/dma. v38i0.45358

Chuenpagdee, R.; Pascual-Fernández, J. J.; Szeliánszky, E.; Alegret, J. L.; Fraga, J.; Jentoft, S. Marine protected areas: Re-thinking their inception. Marine Policy, 39, 234-240, 2013. doi: 10.1016/j.marpol.2012.10.016

Cicin-Sain, B.; Belfiore, S. Linking marine protected areas to integrated coastal and ocean management: A review of theory and practice. Ocean \& Coastal Management, 48, 847-868, 2005.

Cicin-Sain, B.; Knecht, R. W. Integrated coastal and ocean management: concepts and practices. Washington: Island Press, 1998. 517 p.

CONAMA - Conselho Nacional do Meio Ambiente. Resolução $n^{\circ} 347$, de 10 de setembro de 2004. Dispõe sobre a proteção do patrimônio espeleológico. Publicada no DOU $n^{0} 176$, de 13/09/2004, págs. 54-55.

Conti, B. R.; Irving, M. de A.; Antunes, D. de C. O ICMS-Ecológico e as Unidades de Conservação no Estado do Rio de Janeiro. Desenvolvimento e Meio Ambiente, 35, 241-258, 2015. doi: 0.5380/dma.v35i0.41204

Diegues, A. C. S. O mito moderno da natureza intocada. 3. ed. São Paulo: Hucitec, Núcleo de Apoio à Pesquisa sobre Populações Humanas e Áreas Úmidas Brasileiras, USP, 2001. $161 \mathrm{p}$.

Dourojeanni, M. J.; Pádua, M. T. J. Biodiversidade: a hora decisiva. 2. ed. Curitiba: Editora UFPR, 2007. 284 p.

Edgar, G. J. et al. Global conservation outcomes depend on marine protected areas with five key features. Nature, 1-5, 2014. doi: 10.1038 /nature 13022 
Euclydes, A. C. P.; Magalhães, S. R. A. A Área de Proteção Ambiental (APA) e o ICMS Ecológico em Minas Gerais: algumas reflexões. Geografias, 2(2), 39-55, 2006.

Faria, H. H. de. Eficácia de gestão de Unidades de Conservação gerenciadas pelo Instituto Florestal de São Paulo, Brasil. Presidente Prudente, Tese (Doutorado em Geografia, na área de concentração Desenvolvimento Regional e Planejamento Ambiental) - UNESP, 2004.

Faraco, L. F. D.; Andriguetto-Filho, J. M.; Daw, T.; Lana, P. da C.; Teixeira, C. F. Vulnerability Among Fishers in Southern Brazil and its Relation to Marine Protected Areas in a Scenario of Declining Fisheries. Desenvolvimento e Meio Ambiente, 38, 51-76, 2016. doi: 10.5380/dma.v38i0.45850

Ferreira, L. da C. Dimensões humanas da biodiversidade: mudanças sociais e conflitos em torno de Áreas Protegidas no Vale do Ribeira, SP, Brasil. Ambiente \& Sociedade, 7(1), 47-68, 2004.

Gerhardinger, L. C.; Godoy, E. A. S.; Jones, P. J. S.; Sales, G.; Ferreira, B. P. Marine protected dramas: the flaws of the Brazilian National System of Marine Protected Areas. Environmental Management, 47(4), 630-643, 2011. doi: 10.1007/s00267-010-9554-7

Gill, D. A.; Mascia, M. B.; Ahmadia, G. N.; Glew, L.; Lester, S. E.; Barnes, M.; Craigie, I.; Darling, E. S.; Free, C. M.; Geldmann, J.; Holst, S.; Jensen, O. P.; White, A. T.; Basurto, X.; Coad, L.; Gates, R. D.; Guannel, G.; Mumby, P. J.; Thomas, H.; Whitmee, S.; Woodley, S.; Fox, H. E. Capacity shortfalls hinder the performance of marine protected areas globally. Nature, 543, 665-669, 2017. doi: 10.1038/nature21708

Hanazaki, N.; Berkes, F.; Seixas, C.; Peroni, N. Livelihood Diversity, Food Security and Resilience among the Caiçara of Coastal Brazil. Human Ecology, 41, 153-164, 2013.

Hill, L. S.; Johnson, J. A.; Adamowski, J. Meeting Aichi Target 11: Equity considerations in Marine Protected Areas design. Ocean \& Coastal Management, 134, 112-119, 2016. doi: 10.1016/j.ocecoaman.2016.09.017

ICMBio/WWF - Instituto Chico Mendes de Conservação da Biodiversidade, WWF-Brasil. Avaliação comparada das aplicações do método Rappam nas unidades de conservação federais, nos ciclos 2005-06 e 2010. Brasília: ICMBio,
2011.134 p.

Inouye, C. E. N.; Sousa Jr., W. C. de; Freitas, D. M. de; Simões, E. Modelling the spatial dynamics of urban growth and land use changes in the north coast of São Paulo, Brazil. Ocean \& Coastal Management, 108, 147-157, 2015. doi: 10.1016/j.ocecoaman.2014.12.016

IBGE - Instituto Brasileiro de Geografia e Estatística. IBGE Cidades. Disponível em: https://cidades.ibge.gov. br/. Acesso em: ago. 2017.

Kalikoski, D. S.; Seixas, C. S.; Almudi, T. Gestão compartilhada e comunitária da pesca no Brasil: avanços e desafios. Ambiente \& Sociedade, 12(1), 151-172, 2009.

Loitzenbauer, E.; Mendes, C. A. B. A faixa terrestre da zona costeira e os recursos hídricos na Região Hidrográfica do Atlântico Sul, Brasil. Revista da Gestão Costeira Integrada, 14(1), 81-94, 2014. doi: 10.5894/rgci448

Macedo, H. S.; Vivacqua, M.; Rodrigues, H. C. L.; Gerhardinger, L. C. Governing wide coastal-marine protected territories: A governance analysis of the Baleia Franca Environmental Protection Area in South Brazil. Marine Policy, 41, 118-125, 2013. doi: 10.1016/j.marpol.2013.01.008

Machado, I. C.; Nordi, N.; Henriques, M. B.; Cardoso, T. A.; Pereira, O. M. A integração da pesquisa ao conhecimento ecológico local no subsídio ao manejo: variações no estoque natural da ostra de mangue Crassostrea spp. na reserva extrativista do Mandira, Cananéia-SP, Brasil. Ambiente \& Sociedade, 14(1), 1-22, 2011. doi: 10.1590/ S1414-753X2011000100002

Medeiros, R. Evolução das tipologias e categorias de áreas protegidas no Brasil. Ambiente \& Sociedade, 9(1), 41-64, 2006 .

Mello, K. de; Toppa, R. H.; Abessa, D. M. Dinâmica da paisagem do município de Cubatão: crescimento entre portos, indústrias e a Serra do Mar. O Mundo da Saúde, 35(1), 42-46, 2011.

MMA - Ministério do Meio Ambiente. Gerência de Biodiversidade Aquática e Recursos Pesqueiros. Panorama da conservação dos ecossistemas costeiros e marinhos no Brasil. Brasília: MMA/SBF/GBA, 2010. 148 p.

MMA - Ministério do Meio Ambiente. Cadastro Nacional 
de Unidades de Conservação (CNUC). Disponível em: http://www.mma.gov.br/areas-protegidas/cadastro-nacional-de-ucs/. Acesso em: ago. 2017.

Myers, N.; Mittermeier, R. A.; Mittermeier, C. G.; Fonseca, G. A. B. da; Kent, J. Biodiversity hotspots for conservation priorities. Nature, 403, 853-858, 2000.

Oliveira, J. A. P. de. Implementing environmental policies in developing countries through decentralization: the case of Protected Areas in Bahia, Brazil. World Development, 30(10), 1713-1736, 2002.

Oliveira-Júnior, J. G. C.; Ladle, R. J.; Correia, R.; Batista, V. S. Measuring what matters - Identifying indicators of success for Brazilian marine protected areas. Marine Policy, 74, 91-98, 2016. doi: 10.1016/j.marpol.2016.09.018

Pádua, M. T. J. Do Sistema Nacional de Unidades de Conservação. In: Medeiros, R.; Araújo, F. F. S. (Orgs.). Dez anos do Sistema Nacional de Unidades de Conservação da Natureza: lições do passado, realizações presentes e perspectivas para o futuro. Brasília: MMA, 2011. p. 21-36.

Peccatiello, A. F. O. Políticas públicas ambientais no Brasil: da administração dos recursos naturais (1930) à criação do Sistema Nacional de Unidades de Conservação (2000). Desenvolvimento e Meio Ambiente, 24, 71-82, 2011. doi: 10.5380/dma.v24i0.21542

Pellin, A. Avaliação dos aspectos relacionados à criação e manejo de Reservas Particulares do Patrimônio Natural no Estado do Mato Grosso do Sul, Brasil. São Carlos, Tese (Doutorado em Ciências - Programa de Ciências da Engenharia Ambiental) - UFSCar, 2010.

Pellin, A.; Pellin, A.; Scherer, M. E. G. Mosaicos de áreas protegidas criados em território nacional brasileiro e estratégias para a sua gestão. Revista Brasileira de Gestão Ambiental e Sustentabilidade, 4(7), 177-190, 2017. doi: 10.21438/rbgas.040718

Pellin, A.; Ranieri, V. E. L. Voluntary preservation on private land in Brazil: characterization and assessment of the effectiveness of managing Private Reserves of Natural Heritage. Brazilian Geographical Journal: Geosciences and Humanities Research Médium, 7(1), 33-52, 2016.

Pereira, P. E.; Diegues, A. C. Conhecimento de populações tradicionais como possibilidade de conservação da natureza: uma reflexão sobre a perspectiva da etnoconservação. Desenvolvimento e Meio Ambiente, 22, 37-50, 2010. doi: 10.5380/dma.v22i0.16054

Piedade, F. L.; Molina, S. M. G. O estado da arte da legislação aplicável às populações tradicionais: o caso da Unidade de Conservação Juréia-Itatins. OLAM-Ciência e Tecnologia, 1(2), 218-245, 2013.

Pinheiro, M. R. (Org.). Recomendações para reconhecimento e implementação de Mosaicos de Áreas Protegidas. Brasília, DF, GTZ, 2010. 82 p.

Pinto, J. de S.; Frainer, D. M.; Oliveira, A. K. M. de; Souza, C. C. de. Diagnóstico e avaliação da eficiência da preservação do ambiente em Mato Grosso do Sul a partir da inclusão do pagamento de serviços ambientais. Desenvolvimento e Meio Ambiente, 35, 225-240, 2015. doi: 0.5380/ dma.v35i0.41238

Pinto, L. P.; Hirota, M.; Guimarães, E.; Fonseca, M.; Martinez, D. I.; Takahashi, C. K. Unidades de Conservação Municipais da Mata Atlântica. Relatório Técnico. Fundação SOS Mata Atlântica, julho, 2017. 104 p.

Polette, M.; Rebouças, G. N.; Filardi, A. C. L.; Vieira, P. F. Rumo à gestão integrada e participativa de zonas costeiras no Brasil: percepções da comunidade científica e do terceiro setor. Gestão Costeira Integrada, 5, 43-48, 2004.

Prates, A. P.; Blanc, D. (Org.). Áreas aquáticas protegidas como instrumento de gestão pesqueira. Brasília: MMA/ SBF, 2007.

Rainho, A. P. Desvendando a assimetria entre os programas de verdade na construção do Plano de Manejo: estudo de caso no Parque Nacional de Superagui. Tessituras, 2(2), 269-292, 2014.

Reis, A. F.; Queiroz, O. T. M. M. Concessões nas Unidades de Conservação do Estado de São Paulo: reflexões, oportunidades e desafios. Revista Brasileira de Ecoturismo, 10(2), 376-387, 2017.

Rodrigues, L. M.; Magro, T. C. Resex Ilha do Tumba, Cananéia/SP: proposta metodológica de avaliação de gestão ambiental. In: Moura, G. G. M. (Org.). Avanços em oceanografia humana: o socioambientalismo nas Ciências do 
Mar. Jundiaí, SP: Paco, 2017. p. 197-232.

Sá, M. P. G. e; Neiman, Z.; Bondioli, A. C. V. O Santuário Ecológico de Ilhabela como área marinha protegida a ser incorporada ao SNUC: panorama atual e próximos passos. Desenvolvimento e Meio Ambiente, 41, 158-173, 2017. doi: 10.5380/dma.v41i0.49117

Sanches, R. A. Caiçara Communities of the Southeastern Coast of São Paulo State (Brazil): Traditional Activies and Conservation Policy for the Atlantic Rain Forest. Human Ecology Review, 8(2), 52-64, 2001.

São Paulo. Lei $n^{\circ} 8.510$, de 29 de dezembro de 1993. Altera a Lei n ${ }^{\circ} 3.201$, de 23 de dezembro de 1981, que dispõe sobre a parcela, pertencente aos municípios, do produto da arrecadação do Imposto sobre Operações Relativas à Circulação de Mercadorias e sobre Prestações de Serviços de Transporte Interestadual e lntemunicipal e de Comunicação - ICMS. São Paulo: DOE de 30-12-93.

São Paulo. Lei $n^{\circ}$ 10.019, de 3 de julho de 1998. Dispõe sobre o Plano Estadual de Gerenciamento Costeiro. São Paulo: Assembleia Legislativa, 1998.

São Paulo. Decreto $n^{\circ}$ 51.150, de 3 de outubro de 2006. Dispõe sobre o reconhecimento das Reservas Particulares do Patrimônio Natural, no âmbito do Estado de São Paulo, institui o Programa Estadual de Apoio às Reservas Particulares do Patrimônio Natural e dá providências correlatas. São Paulo: Assembleia Legislativa, 2006.

São Paulo. Decreto $n^{\circ}$ 51.453, de 29 de dezembro de 2006. Cria o Sistema Estadual de Florestas - SIEFLOR e dá providências correlatas. Publicado na Casa Civil, aos 29 de dezembro de 2006.

São Paulo. Lei $n^{\circ} 12.810$, de 21 de fevereiro de 2008. Altera os limites do Parque Estadual de Jacupiranga, criado pelo Decreto-lei n. 145, de 8 de agosto de 1969, e atribui novas denominações por subdivisão, reclassifica, exclui e inclui áreas que especifica, institui o Mosaico de Unidades de Conservação do Jacupiranga e dá outras providências. São Paulo: Assembleia Legislativa, 2008a.

São Paulo. Decreto $n^{\circ} 53.528$, de 8 de outubro de 2008. Cria o Mosaico das Ilhas e Áreas Marinhas Protegidas do Litoral Paulista, e dá providências correlatas. São Paulo: Assembleia Legislativa, 2008b.
São Paulo. Decreto ${ }^{\circ} 54.079$, de 4 de março de 2009. Altera os artigos $5^{\circ}, 6^{\circ}$ e $9^{\circ}$, acrescenta o artigo $9^{\circ} \mathrm{A}$ e modifica os Anexos do Decreto $\mathrm{n}^{\circ}$ 51.453, de 29 de dezembro de 2006, que cria o Sistema Estadual de Florestas - SIEFLOR e dá providências correlatas. Publicado na Casa Civil, aos 4 de março de 2009.

São Paulo. Lei $n^{\circ} 14.982$, de 8 de abril de 2013. Altera os limites da Estação Ecológica da Juréia-Itatins, na forma que especifica, e dá outras providências. São Paulo: Assembleia Legislativa, 2013.

São Paulo. Decreto $n^{\circ}$ 60.302, de 27 de março de 2014. Institui o Sistema de Informação e Gestão de Áreas Protegidas e de Interesse Ambiental do Estado de São Paulo - SIGAP e dá providências correlatas. São Paulo: Publicado na Casa Civil, aos 27 de março de 2014.

Scherer, M.; Sanches, M.; Negreiros, D. H. de. Gestão das zonas costeiras e as políticas públicas no Brasil: um diagnóstico. In: Barragán Muñoz, J. M. (Coord.). Manejo Costero Integrado y Política Pública em Iberoamérica: Un diagnóstico. Necesidad de Cambio. Red IBERMAR (CYTED), Cádiz, 380 p., 2010.

Silva, M. The Brazilian Protected Areas Program. Conservation Biology, 19(3), 608-611, 2005.

SMA - Secretaria do Meio Ambiente do Estado de São Paulo. Meio ambiente paulista: relatório de qualidade ambiental 2015. Secretaria do Meio Ambiente do Estado de São Paulo, Coordenadoria de Planejamento Ambiental. Organizador Edgar Cesar de Barros. 1 ed. São Paulo: SMA, 2015.

Trimble, M.; Araújo, L. G. de; Seixas, C. S. One party does not tango! Fishers' non-participation as a barrier to co-management in Paraty, Brazil. Ocean \& Coastal Management, 92, 9-18, 2014. doi: 10.1016/j.ocecoaman.2014.02.004

Vivacqua, M.; Vieira, P. F. Conflitos socioambientais em Unidades de Conservação. Política \& Sociedade, 7, 139$162,2005$. 